\title{
Therapie des hepatozellulären Karzinoms - eine neue Dekade?
}

Das hepatozelluläre Karzinom (HCC) ist der häufigste primäre Lebertumor und gehört weltweit betrachtet zu den Karzinomen mit der am stärksten steigenden Inzidenz. Der überwiegende Teil dieser Tumore entsteht vor dem Hintergrund einer chronischen hepatischen Inflammation und dem konsekutiven zirrhotischen Umbau der Leber. In Europa sind die alkoholassoziierte Leberzirrhose (ca. 50\%), gefolgt von der chronischen Hepatitis C (ca. $25 \%$ ), die häufigsten Ursachen. In den letzten Jahren ist jedoch eine eindrucksvolle Zunahme der Entstehung von HCCs auf dem Boden von nichtalkoholischen Fettlebererkrankungen (NAFLD) bzw. der nichtalkoholischen Steatohepatitis (NASH) zu verzeichnen [1]. Trotz der Empfehlung zu einer regelmäßigen Überwachung (Surveillance) von Risikopatient*innen wird das HCC häufig erst in intermediären oder fortgeschrittenen Stadien, gemäß der Barcelona Clinic of Liver Cancer Classification (BCLC), diagnostiziert. Eine potenziell kurative Behandlungsstrategie, d.h. Resektion, Lebertransplantation oder Ablation (z. B. Radiofrequenz-/Mikrowellenablation oder Brachytherapie), ist allerdings nur in frühen Krankheitsstadien möglich [2]. Für fortgeschrittene Krankheitsstadien (d.h. ausgedehnten Tumorbefall in der Leber, maligne Pfortaderinfiltration, extrahepatische Metastasen) sind mit der Einführung der Immuntherapie, d.h. der kombinierten Systemtherapie unter Einbeziehung eines Immuncheckpoint-Inhibitors, neue Behandlungsoptionen verfügbar, sodass tatsächlich von einer „neuen Dekade“ in der HCC-Therapie gesprochen werden kann [3].

Das Verständnis über den sequenziellen Einsatz mehrerer Therapielinien sowie die Etablierung der Immuntherapie konnte die Prognose des HCC im BCLC-Stadium C in jüngster Zeit signifikant verbessern. Tyrosinkinase-Inhibitoren (TKI) waren über lange Zeit die einzig verfügbare Therapieoption mit teils schwer beherrschbaren Nebenwirkungsprofilen und insgesamt begrenzter Wirksamkeit. Im Vergleich zu den TKI konnte der Einsatz von Immun-Checkpoint-Inhibitoren (ICI) höhere und längere Tumoransprechraten erzielen [3].

Trotz der vielversprechenden Ausgangsdatenlage verliefen die beiden initialen Phase-III-Studien, die den Einsatz der Im- muntherapie beim HCC prüften, negativ. Sowohl Nivolumab gegen Sorafenib als Erstlinientherapie (Studie CheckMate 459), als auch Pembrolizumab gegen best supportive care (BSC) beim vortherapierten HCC (Studie Keynote-240) konnten zwar die primären Endpunkte Gesamtüberleben (OS) und progressionsfreies Überleben (PFS) tendenziell verbessern, erreichten jedoch die vordefinierte statistische Signifikanz nicht. Nichtsdestotrotz konnte der Einsatz der Immuntherapie beim HCC ungewohnt hohe Tumorremissionsraten erbringen [4].

Die Überlegung, verschiedene antitumorale Mechanismen im Sinne einer T-ZellAktivierung sowie einer Antiangiogenese synergistisch auszunutzen, führte zum kombinierten Einsatz des programmed death ligand 1 (PDL-1) Inhibitors Atezolizumab und des anti vascular endothelial growth factor A (VEGF-A) Bevacizumab. Auf dem Boden der Ergebnisse der PhaseIII-IMbrave150-Studie wurde diese Kombinationstherapie als systemische HCCErstlinientherapie im November 2020 von der European Medicines Agency (EMA) zugelassen. Es war das erste Therapieregime,

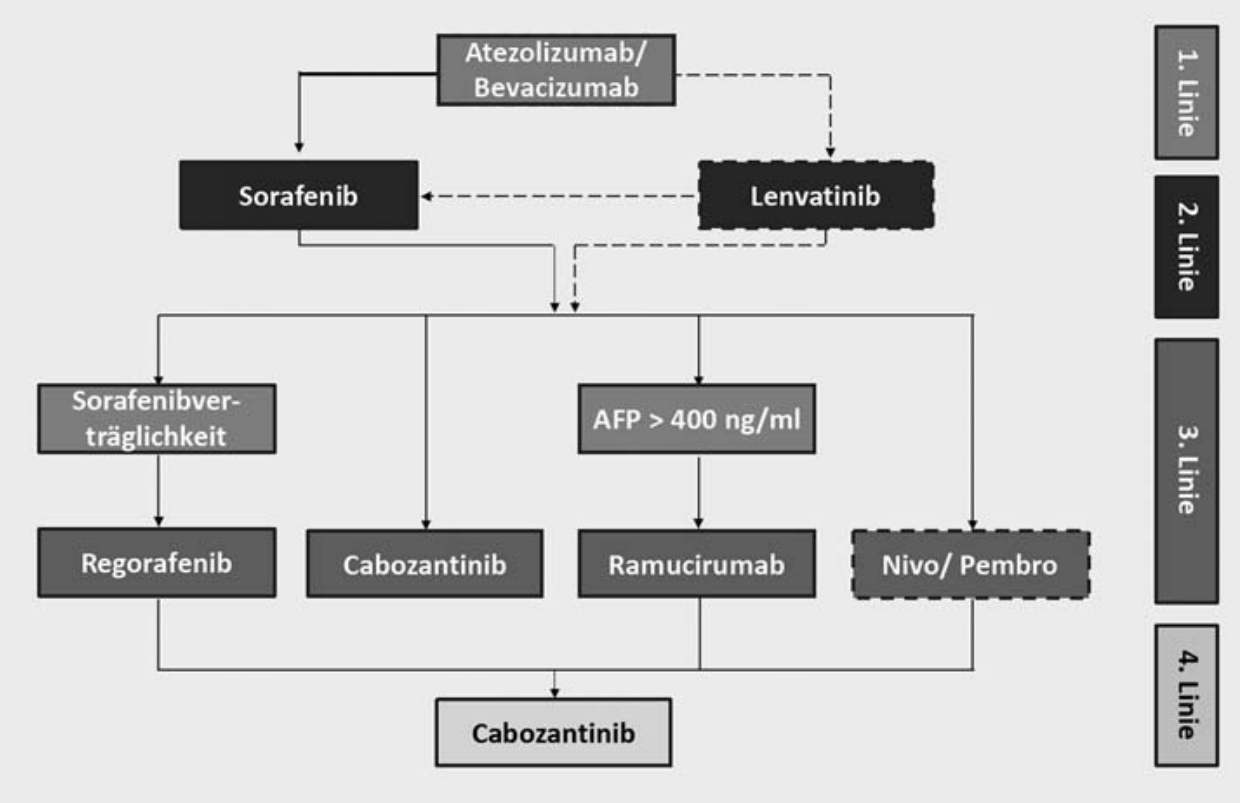

- Abb. 1 Vorschlag der systemischen Sequenztherapie für das hepatozelluläre Karzinom im BCLC-Stadium C. Die neue Erstlinientherapie besteht aus Atezolizumab (anti-PDL-1) und Bevacizumab (anti-VEGF). Die gestrichelten Linien zeigen Varianten außerhalb der aktuellen Zulassung der Substanzen an. 
das gegenüber dem TKI Sorafenib einen signifikanten Überlebensvorteil mit einer Hazard Ratio (HR) von 0,66 $(95 \% \mathrm{Cl} 0,52-$ $0,85 ; p=0,0009)$ erzielen konnte. Neben einem längeren Gesamtüberleben (OS: 19,2 vs. 13,4 Monate) und progressionsfreien Überleben (PFS: 6,9 vs. 4,3 Monate) sowie höheren Tumoransprechraten in diesen fortgeschrittenen Tumorstadien konnten niedrigere Toxizitätsraten, eine bessere Verträglichkeit sowie eine Verlängerung des Intervalls bis zur Lebensqualitätsverschlechterung erreicht werden [5]. Der Überlebensvorteil der Kombinationstherapie erstreckt sich über alle Subgruppen hinweg, mit Betonung auf denjenigen $\mathrm{HCC}$, die auf dem Boden einer viral induzierten Leberzirrhose entstanden sind. Rezente präklinische Hinweise über eine verminderte Wirksamkeit bei nichtalkoholischer Steatohepatitis (NASH)-assoziierten HCC müssen in zukünftigen klinischen Studien adressiert werden [6]. Einem (nicht signifikant) höheren Anteil an gastrointestinalen Blutungskomplikationen unter Atezolizumab/Bevacizumab sollte im klinischen Alltag mit einer strengen endoskopischen Varizenkontrolle und -therapie begegnet werden [4]. Der Einsatz von Immuntherapie ist besonders im post-Lebertransplantations-Setting (Gefahr einer IClinduzierten Organabstoßung) und bei vorbestehenden unkontrollierten Autoimmunerkrankungen (Gefahr eines ICl-induzierten Schubes) zu vermeiden.

Systemische Therapieoptionen nach dem Versagen von Atezolizumab/Bevacizumab müssen aktuell neu definiert werden. Substanzen, welche für eine Second-line-Therapie zur Verfügung stehen, wurden allesamt nur nach Sorafenibversagen bzw. -intoleranz getestet. Eine Rationale für den Einsatz von TKI nach dem Versagen von Atezolizumab/Bevacizumab ist bei erhaltener Leberfunktion klar gegeben, eine Evidenz über deren Effektivität besteht allerdings nur für ihren Einsatz im Erstliniensetting bzw. nach Sorafenibversagen. PhaseIII-Daten zur Wahl einer Sequenztherapie fehlen aktuell. Es erscheint daher gerechtfertigt, den Einsatz aller zugelassenen Substanzen nach ICI-Versagen zu befürworten [7]. Ein Verschieben der ehemals etablierten Sequenzen ist in $\mathbf{A}$ Abb. 1 skizziert.
Verschiedene neue Kombinationen systemischer Therapien (z.B. ICI + TKI oder anti-PD1/PDL-1 + anti-CTLA4) mit vielversprechenden Phase-I/II-Daten werden aktuell in Phase-III-Studien getestet. Daneben zeichnet sich aber auch ab, dass sich die Indikationen zur Systemtherapie in Zukunft erweitern werden. Die verhältnismäßig hohen Ansprechraten unter ICI-basierten Regimen bilden die Rationale für einen (neo)adjuvanten Einsatz der Systemtherapie jenseits des BCLC-C-Stadiums [8]. Ob dies eine angemessene Strategie ist, den hohen Rezidivraten nach Resektion bzw. Lokaltherapie zu begegnen, wird aktuell ebenfalls in unterschiedlichsten Phase-III-Studien untersucht [9].

Trotz der Euphorie über die rezenten Erfolge der Systemtherapie darf nicht vergessen werden, dass ca. ein Viertel der Patienten nicht auf $\mathrm{ICl}$-basierte Therapien anspricht und das mittlere PFS unter Atezolizumab/Bevacizumab lediglich bei $6,8 \mathrm{Mo-}$ naten liegt. Bis dato ist es nicht gelungen, verlässliche Biomarker, die ein Therapieansprechen vorhersagen können, zu identifizieren [4]. Vor allem die Heterogenität des Tumormikroenvironments je nach Ätiologie der zugrundeliegenden Leberschädigung [10] sowie die Leberfunktionseinschränkung per se stellen die größten Hürden bei der Weiterentwicklung der pharmakologischen Therapie des HCCs dar - und sind damit wichtige Herausforderungen für die neue Dekade der HCCBehandlung.

\section{Interessenkonflikt}

Forschungsarbeiten im Labor von Prof. Tacke wurden durch die Firmen Gilead, BMS, Allergan und Inventiva gefördert.

Raphael Mohr gibt an, dass kein Interessenkonflikt besteht.

\section{Autoren}

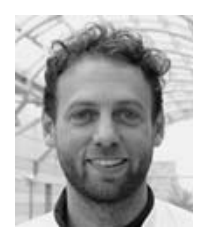

\section{Raphael Mohr}

Charité - Universitätsmedizin Berlin, Medizinische Klinik m. S. Hepatologie und Gastroenterologie, Campus VirchowKlinikum (CVK) und Campus Charité Mitte (CCM)

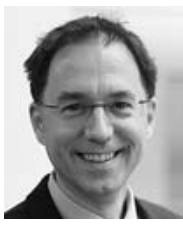

\section{Frank Tacke}

Charité - Universitätsmedizin Berlin, Medizinische Klinik m. S. Hepatologie und Gastroenterologie, Campus VirchowKlinikum (CVK) und Campus Charité Mitte (CCM)

\section{Korrespondenzadresse}

Prof. Dr. med. Frank Tacke

Charité - Universitätsmedizin Berlin

Medizinische Klinik m. S. Hepatologie und Gastroenterologie, Campus Virchow-Klini-

kum (CVK) und Campus Charité Mitte (CCM)

Augustenburger Platz 1

10117 Berlin

Deutschland

frank.tacke@charite.de

\section{Literatur}

[1] Lee YT, Wang J], Luu M et al. The mortality and overall survival trends of primary liver cancer in the United States. J Natl Cancer Inst 2021. Epub ahead of print. doi:10.1093/jnci/ djab079

[2] Forner A, Reig M, Bruix J. Hepatocellular carcinoma. Lancet 2018; 391: 1301-1314

[3] Roderburg C, Ozdirik B, Wree A et al. Systemic treatment of hepatocellular carcinoma: from sorafenib to combination therapies. Hepat Oncol 2020; 7: HEP20

[4] Mohr R, Jost-Brinkmann F, Ozdirik B et al. Lessons from immune checkpoint inhibitor trials in hepatocellular carcinoma. Front Immunol 2021; 12: 652172

[5] Finn RS, Qin S, Ikeda M et al. Atezolizumab plus bevacizumab in unresectable hepatocellular carcinoma. N Engl J Med 2020; 382: 1894-1905

[6] Pfister D, Nunez NG, Pinyol R et al. NASH limits anti-tumour surveillance in immunotherapy-treated HCC. Nature 2021; 592: 450-456

[7] Vogel A, Martinelli E, clinicalguidelines@esmo.org EGCEa, Committee EG. Updated treatment recommendations for hepatocellular carcinoma (HCC) from the ESMO Clinical Practice Guidelines. Ann Oncol 2021; 32: 801-805

[8] Greten TF, Mauda-Havakuk M, Heinrich B et al. Combined locoregional-immunotherapy for liver cancer. J Hepatol 2019; 70: 9991007

[9] Forner A, Da Fonseca LG, Diaz-Gonzalez A et al. Controversies in the management of hepatocellular carcinoma. JHEP Rep 2019; 1: 17-29

[10] Peiseler M, Tacke F. Inflammatory mechanisms underlying nonalcoholic steatohepatitis and the transition to hepatocellular carcinoma. Cancers (Basel) 2021; 13: 730 